\title{
Atypical Bilateral Fuchs Uveitis: Diagnostic Challenges
}

Cristóbal Couto $^{\mathrm{b}}$ Erika Hurtado $^{\mathrm{b}}$ Dana Faingold ${ }^{\mathrm{a}}$ Carmen Demetrio $^{\mathrm{b}}$ Ariel Schlaen $^{\mathrm{b}}$ Marcelo Zas ${ }^{\mathrm{b}}$ Jorge Zarate ${ }^{c}$ Silvia Rosetti ${ }^{\mathrm{d}}$ Andrea Paes de Lima ${ }^{c}$ Juan Oscar Croxatto ${ }^{d}$ Pablo Chiaradía ${ }^{b}$ Miguel N. Burnier ${ }^{a}$

${ }^{a}$ Ocular Pathology Laboratory, Department of Ophthalmology, McGill University, Montréal, Qué.; ${ }^{b}$ Uveitis, Retina and Cornea Clinics, Department of Ophthalmology, University of Buenos Aires, 'Department of Pathology, University of Buenos Aires, and ${ }^{\mathrm{d}}$ Microbiology and Pathology Laboratory, Fundación Oftalmológica Argentina, Buenos Aires, Argentina

\section{Key Words}

Bilateral Fuchs uveitis · In vivo confocal microscopy $\cdot$ Diagnosis

\section{Abstract}

Bilateral Fuchs uveitis associated with vitreous infiltration and posterior segment involvement requires a thorough diagnostic evaluation. The lack of well-defined diagnostic criteria makes identification of this entity difficult. The aim of this case report was to present the characteristics of a patient with atypical Fuchs uveitis and the procedures needed to rule out the differential diagnosis with specific attention to the utility of in vivo confocal microscopy (IVCM). Case Report: One case of chronic bilateral uveitis with severe vitreous opacities is presented. After extensive systemic workup, including vitrectomy, the case had no identifiable systemic etiology. IVCM of the cornea revealed the presence of dendritiform keratic precipitates. Conclusion: The diagnosis of Fuchs uveitis is based on clinical findings as no confirmatory laboratory tests are available. A high index of suspicion is key to an early diagnosis, especially in the cases with vitreous opacities and posterior segment manifestations. Auxiliary tests such as IVCM may aid the clinician in the diagnosis of Fuchs uveitis.

(C) 2015 S. Karger AG, Basel 
Couto et al:: Atypical Bilateral Fuchs Uveitis: Diagnostic Challenges

Fuchs heterochromic uveitis (FU) represents $<6 \%$ of all cases of uveitis [1]. It is characterized by low-grade intraocular inflammation, iris heterochromia or atrophy or both, characteristic keratic precipitates (KPs) distributed all over the endothelium, absence of synechiae, development of cataract and, less frequently, of glaucoma [2]. The aim of this case report was to present the characteristics of a patient with atypical FU and the procedures needed to rule out differential diagnosis with specific attention to the utility of in vivo confocal microscopy (IVCM).

A 67-year-old woman presented to the uveitis clinic with a 2-year history of decreased blurred vision in both eyes (OU) without pain or redness. She had had rheumatoid arthritis for 3 years and had been treated with methylprednisolone $30 \mathrm{mg} /$ day and methotrexate 10 $\mathrm{mg} /$ week. Her ocular medical history was bilateral chronic anterior uveitis. On examination, the best corrected visual acuity was 20/30 in her right eye and 20/40 in her left eye (OS). IOP was $14 \mathrm{~mm} \mathrm{Hg}$ in OU. A slit lamp examination of OU revealed diffuse stellate medium and little KPs (fig. 1), 1+ cells and no flare in the anterior chamber and smooth diffuse parenchymal iris atrophy without posterior synechiae. The fundus examination revealed $3+$ of vitreous haze in OU in the absence of cataract (fig. 2). Optical coherence tomography could not asses the retina due to vitreous haze, and the fluorescein angiography did not show any posterior inflammatory signs. The workup for infectious uveitis was negative. Sarcoidosis was ruled out with chest imaging (chest CT scan) and laboratory tests (tuberculin skin test and serum angiotensin-converting enzyme).

The patient was diagnosed with bilateral FU based on the diagnostic criteria [3]. However, due to the age of the patient and the severity of the vitreous haze despite having received extensive treatment with corticosteroids and immunosuppressive agents, we attempted to rule out a second diagnosis: primary ocular lymphoma. Neurological evaluation did not reveal any underlying disease, and the MRI and cerebrospinal fluid analysis were normal. A diagnostic vitrectomy was performed in her OS without complications. The cytopathological study showed isolated polymorphonuclear cells, lymphocytes, histiocytes, erythrocytes and amorphous material (fig. 3). Flow cytometry showed B cells (92\%), without signs of atypia (atypical cells). The diagnosis of lymphoma was ruled out. The patient improved her visual acuity to 20/20 (OS) without signs of anterior uveitis or vitreous haze. Due to vitreous opacity in her right eye, a vitrectomy was performed in order to improve the symptoms and for diagnostic purposes. The cytopathological study was negative for lymphoma, and PCR was negative for HSV, HZV, CMV, toxoplasmosis and rubella. The patient recovered her visual acuity to $20 / 20$ in OU without recurrence of the vitreous haze and anterior uveitis OU. In order to reinforce the clinical diagnosis of FU, we performed an IVCM evaluation of both corneas. The study revealed isolated dendritic cells at the basal membrane and stromal hyperreflective deposits. The endothelium showed polymegethism and numerous cells with long and spider web extensions and cell clusters that could correspond to cells with hyperchromatic round nuclei (lymphocytes) and hyperreflective punctate deposits that could correspond to pigmented cells (melanosomes). Mean endothelial cell density was lower in both corneas. The findings were highly consistent with FU.

FU is a chronic anterior uveitis characterized by a low-grade intraocular inflammation. Chronic anterior uveitis has been associated with noninfectious systemic inflammatory conditions, such as sarcoidosis, juvenile idiopathic arthritis and tubulointerstitial, nephritis and uveitis syndrome, as well as Fuchs heterochromic iridocyclitis associated with rubella infection and systemic infections, such as syphilis and tuberculosis [4].

The condition can also affect the posterior segment with vitreous opacities and disc and retinal capillary leakage in the absence of cystoid macular edema [5-7]. FU is mostly unilat- 
eral, but bilateral involvement was reported in approximately $10-20 \%$ of cases [6]. In one case series, bilateral FU cases had a more aggressive course with more complications [8].

Only $10-50 \%$ of FU cases are identified at initial presentation and, in the absence of classical clinical findings, this syndrome may lead to diagnostic delays lasting years [7]. The etiology and pathogenic mechanisms of FU remain unknown, but herpes simplex, ocular toxoplasmosis, toxocariasis, sarcoidosis, cytomegalovirus anterior uveitis and rubella infection have been implicated in the pathogenesis of this disease [5, 7, 9-11]. KPs can now be studied by noninvasive procedures such as IVCM. Several authors have described KPs in FU using IVCM, and it has been suggested that their morphological appearance may become a diagnostic tool for this type of uveitis. Labbé et al. [12] in a series of 13 patients, found that dendritiform KPs are very characteristic for FU . More recently, Mocan et al. [13] analyzed their IVCM findings in 40 patients with FU and reported that these eyes are characterized by dendritiform KPs which are associated with altered endothelial cell density and morphology.

Bilateral FU associated with vitreous infiltration and posterior segment manifestations requires a diagnostic evaluation. Bouchenaki and Herbort [14] reported vitreous opacities in $97.4 \%$ of their FU patients. In more than $70 \%$ of the misdiagnosed cases, FU was significantly underdiagnosed most likely because of the presence of vitreous infiltration which was not commonly recognized as associated with FU. Sometimes, an extensive evaluation is indicated if there is bilateral uveitis and vitritis with or without neurological symptoms to rule out any other systemic causes like primary intraocular lymphoma. Vitreous opacification may cause severe decrease in visual acuity and several times require pars plana vitrectomy with few complications [15].

The current case report highlights that the clinical picture plays an important role in the diagnosis. More attention should be focused on posterior segment involvement such as vitreous infiltration. In such cases, pars plana vitrectomy should be performed to improve visual acuity, reduce inflammation and rule out malignancies. Several etiologies have been suggested for FU, and a greater understanding of the natural history of this type of uveitis could significantly influence the approach to diagnosis. Determining uniform diagnostic criteria and further investigation of clinical variations of FU with IVCM can help in the differential diagnosis.

\section{Statement of Ethics}

The present case report was conducted according to the ethical principles in medical research involving human subjects stated in the Declaration of Helsinki. The participating individual gave informed consent, and the ethics committee was notified of the case report.

\section{Disclosure Statement}

None of the authors has a conflict of interest in connection with the submission of this case report. 
Couto et al.: Atypical Bilateral Fuchs Uveitis: Diagnostic Challenges

\section{References}

1 Kazokoglu H, Onal S, Tugal-Tutkun I, et al: Demographic and clinical features of uveitis in tertiary centers in Turkey. Ophthalmic Epidemiol 2008;15:285-293.

2 La Hey E, de Jong PT, Kijlstra A: Fuchs heterochromic cyclitis: review of the literature on the pathogenetic mechanisms. Br J Ophthalmol 1994;78:307-312.

3 Mohamed Q, Zamir E: Update on Fuchs' uveitis syndrome. Curr Opin Ophthalmol 2005;16:356-363.

4 Birnbaum A, Little D, Tessler H, Goldstein D: Etiologies of chronic anterior uveitis at a tertiary referral center over 35 years. Ocul Immunol Inflamm 2011;19:19-25.

5 La Hey E, Baarsma GS, de Vries J, Kijlstra A: Clinical analysis of Fuchs heterochromic cyclitis. Doc Ophthalmol 1991;78:225-235.

6 Tugal-Tutkun I, Guney-Tefekli E, et al: A cross-sectional and longitudinal study of Fuchs uveitis syndrome in Turkish patients. Am J Ophthalmol 2009;148:510-515.

7 Al-Mansour YS, Al-Rajhi AA, Al-Dhibi H, Abu El-Asrar AM: Clinical features and prognostic factors in Fuchs uveitis. Int Ophthalmol 2010;30:501-509.

-8 Norrsell K, Sjodell L: Fuchs' heterochromic uveitis: a longitudinal clinical study. Acta Ophthalmol 2008;86:58-64.

-9 Teyssot N, Cassoux N, Lehoang P, Bodaghi B: Fuchs heterochromic cyclitis and ocular toxocariasis. Am J Ophthalmol 2005;139:915-916.

-10 Birnbaum AD, Tessler HH, Schultz K, Farber M, Gao W, Lin P, Oh F, Goldstein D: Epidemiologic relationship between Fuchs heterochromic iridocyclitis and the United States rubella vaccination program. Am J Ophthalmol 2007;144:424-428.

11 Chee SP, Jap A: Presumed Fuchs heterochromic iridocyclitis and Posner-Schlossman syndrome: comparison of cytomegalovirus-positive and negative eyes. Am J Ophthalmol 2008;146:883-889.

$\checkmark 12$ Labbé A, Dupas B, Offret H, Baudouin C, Labetoulle M: Evaluation of keratic precipitates and corneal endothelium in Fuchs' heterochromic cyclitis by in vivo confocal microscopy. Br J Ophthalmol 2009;93:673677.

13 Mocan MC, Kadayifcilar S, Irkeç M: In vivo confocal microscopic evaluation of keratic precipitates and endothelial morphology in Fuchs' uveitis syndrome. Eye (Lond) 2012;26:119-125.

14 Bouchenaki N, Herbort C: Fuchs uveitis: failure to associate vitritis and disc hyperfluorescence with the disease is the major factor for misdiagnosis and diagnostic delay. Middle East Afr J Ophthalmol 2009;16:239-244.

15 Waters FM, Goodall K, Jones N, McLeod D: Vitrectomy for vitreous opacification in Fuchs' heterochromic uveitis. Eye (Lond) 2000;14:216-218.

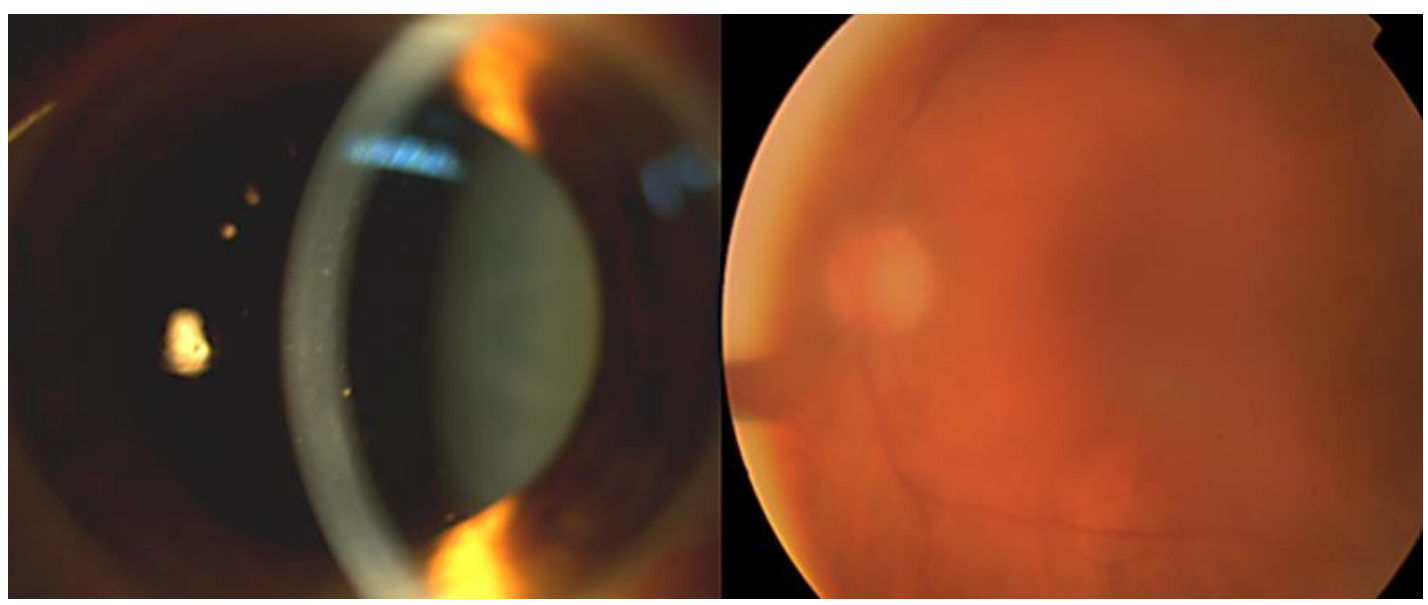

Fig. 1. Diffuse stellate medium, little KPs and vitreous haze in OU. 
Couto et al.: Atypical Bilateral Fuchs Uveitis: Diagnostic Challenges

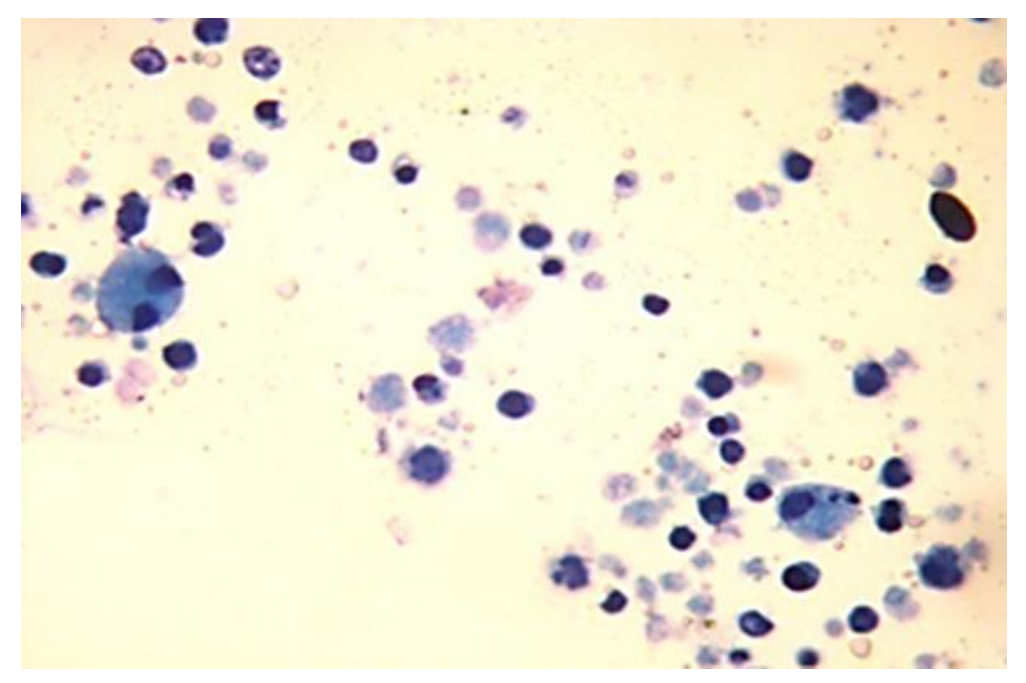

Fig. 2. Histopathological features of the OS.

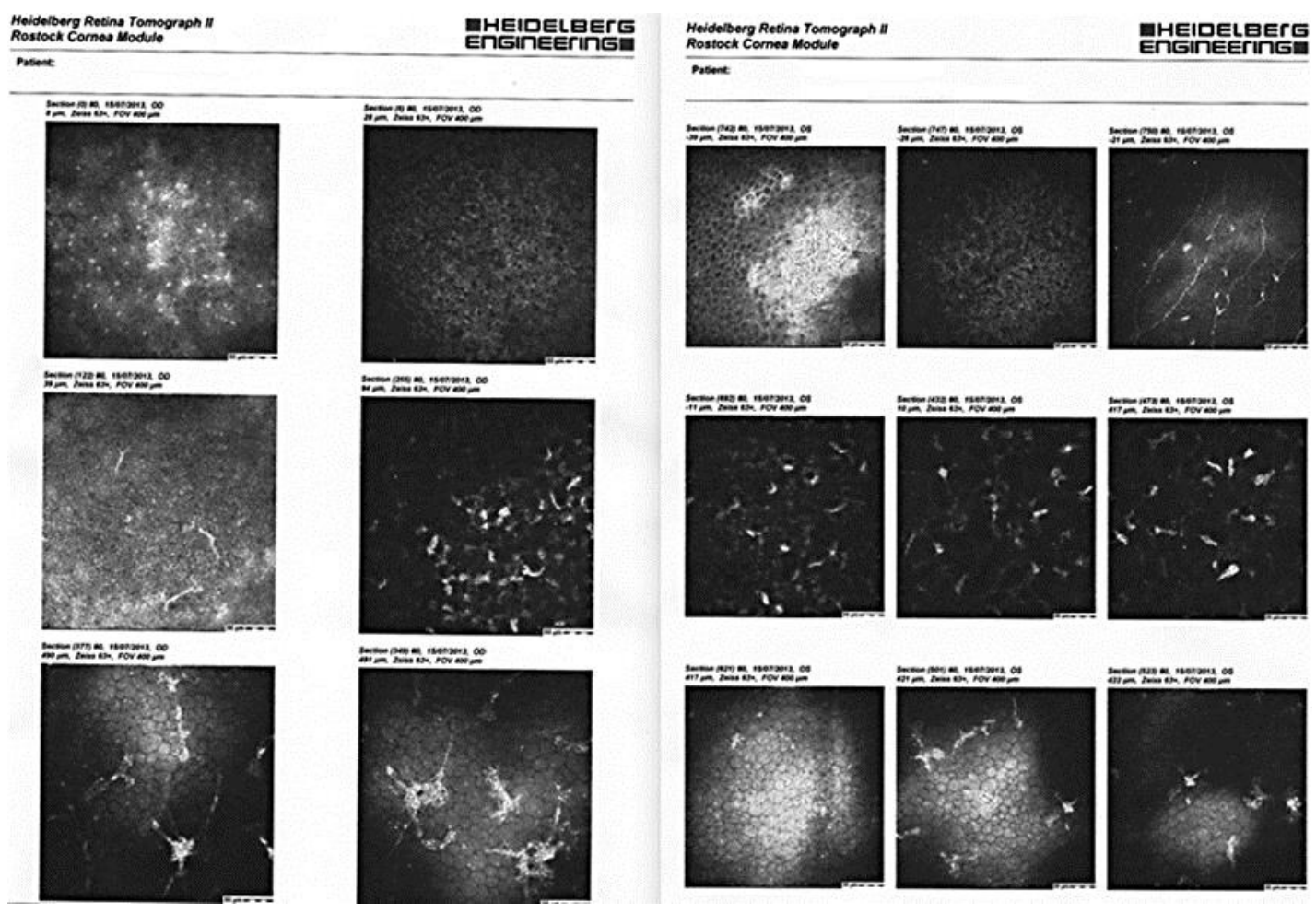

Fig. 3. IVCM features of OU. 